\title{
Enhanced images for checked and carry-on baggage and cargo screening
}

\author{
Glenn Woodell ${ }^{\mathrm{a}}$, Zia-ur Rahman ${ }^{\mathrm{b}}$, Daniel J. Jobson ${ }^{\mathrm{a}}$, Glenn Hines ${ }^{\mathrm{a}}$ \\ ${ }^{a}$ NASA Langley Research Center, Hampton, VA \\ ${ }^{\mathrm{b}}$ College of William and Mary, Williamsburg, VA
}

\begin{abstract}
The current X-ray systems used by airport security personnel for the detection of contraband, and objects such as knives and guns that can impact the security of a flight, have limited effect because of the limited display quality of the X-ray images. Since the displayed images do not possess optimal contrast and sharpness, it is possible for the security personnel to miss potentially hazardous objects. This problem is also common to other disciplines such as medical Xrays, and can be mitigated, to a large extent, by the use of state-of-the-art image processing techniques to enhance the contrast and sharpness of the displayed image. The NASA Langley Research Center's Visual Information Processing Group has developed an image enhancement technology that has direct applications to this problem of inadequate display quality. Airport security X-ray imaging systems would benefit considerably by using this novel technology, making the task of the personnel who have to interpret the X-ray images considerably easier, faster, and more reliable. This improvement would translate into more accurate screening as well as minimizing the screening time delays to airline passengers. This technology, Retinex, has been optimized for consumer applications but has been applied to medical X-rays on a very preliminary basis. The resultant technology could be incorporated into a new breed of commercial x-ray imaging systems which would be transparent to the screener yet allow them to see subtle detail much more easily, reducing the amount of time needed for screening while greatly increasing the effectiveness of contraband detection and thus public safety.
\end{abstract}

Keywords: Retinex, X-rays, image enhancement, baggage screening

\section{INTRODUCTION}

Terrorist activities over the past years and, more recently, the attacks of September 11, 2001 have forced the entire world to better ensure the safety of its people traveling in all modes of transportation. But no mode of transportation is as attractive to terrorist activity as that of air transport. Several terrorist activities prompted the FAA to mandate that air carriers screen all passengers and their carry-on baggage for weapons and other hazardous items. Metal detectors have been required since 1973. Two years after the bombing of Pan Am Flight 103 over Lockerbie, Scotland in 1988, President George H. Bush signed into law, the Aviation Security Improvement Act of 1990 which placed the responsibility of air travel security in the hands of the federal government. Ever since, sophisticated methods of metal and hazardous material detection have been employed in airports throughout the world. Every passenger on a commercial aircraft knows that they will now be thoroughly screened for potential weapons before boarding. Along with the process of this intensive screening come the sometimes long lines of delay before boarding. Most X-ray imaging systems today employ some method of image enhancement to increase the brightness and contrast, and thus the visibility, of the acquired image and even differentiate between organic and metallic substances. However, these methods are not sophisticated enough to handle the wide dynamic ranges that today's X-ray imagers are capable of producing. The Retinex is one such method which has been proven to handle these wide dynamic range images.

Contact: GAW: g.a.woodell@larc.nasa.gov; ZR: zrahman@as.wm.edu; DJJ, d.j.jobson@larc.nasa.gov; GDH: g.d.hines@larc.nasa.gov 


\section{THE RETINEX}

Coined from the combination of retina and cerebral cortex, Edwin Land ${ }^{1}$ first conceived the retinex as a model for human perception of lightness and color perception. This model was extended by Jobson et. $\mathrm{al}^{3,2}$ as a general purpose image enhancement algorithm that provides simultaneous dynamic range compression and color constancy. Dynamic range compression refers to the capability of the algorithm to encompass wide dynamic ranges - scenes that contain both bright and dark regions - without saturating the high intensity values or clipping the low intensity values. Color constancy refers to the ability of the algorithm to produce an output color that is independent of the color of the illuminant: a grey object appears to be grey whether it is viewed under sunlit conditions or under neon lights. Both of these properties are useful in providing better visibility in the presence of illumination changes and shadows. Though Jobson et al.'s Retinex is an attempt to mimic the dynamic range compression and color constancy capabilities of the human visual system, unlike Land's Retinex, it was not developed as a model to explain the functionality of the human visual system. Rather, is a tool for accurate reproduction of imagery acquired by either analog or digital means in situations where the scene lighting varies and/or atmospheric conditions hinder good imagery. However, this version of the Retinex could not provide good tonal rendition so the resulting images often appeared to have a grayish overtone. A multi-scale version of the Retinex ${ }^{3,2}$, the Multiscale Retinex (MSR), was developed ${ }^{2}$ to alleviate these shortcomings of the original Retinex. In addition to its general image enhancement capabilities, the MSR is capable of providing betterthan-observed ${ }^{4}$ imagery, especially where scene content is greatly obscured, as in the case of rain, fog, or severe haze. Although the MSR has primarily been applied to conventional multi-spectral image sources such as color and infrared sensors, it is just as effective on non-conventional, computational imagery such as grayscale image representations of traditional X-rays.

More recently, the Retinex has been used primarily in support of NASA's aviation safety program. The objective in this work is to develop the capability for helping pilots see in inclement weather by enhancing infrared as well as threecolor, visible imagery. These new optimizations to the Retinex technology have far-reaching applications beyond aviation safety, and include areas such as forensic investigations, medical radiography ${ }^{5}$, and consumer imaging. This technology is already available for consumer use in a general purpose software package, from TruView Imaging Company, called PhotoFlair ${ }^{\circledast}$. It was through the testing of this technology on medical X-rays and CT scans that the feasibility of enhancement of airport screening imagery was realized.

\section{CURRENT ART}

In the baggage inspection process, the most crucial element for the detection of prohibited items in passenger baggage is the screener's ability to identify objects from X-ray imagery and classify them as threat or not. The screener's ability to clearly see recognizable shapes and quickly decide whether these are threats or not cannot be overstressed. Although the screener reserves the right to hand search every piece of baggage, this would not be practical for either the passengers who would be subjected to long waiting lines, or for airport operations. The additional time required to clear the passengers to board an airplane would result in fewer flights per day, which would translate into a real financial impact on airport operations and airlines and increase tensions which are already at an all-time high. Within seconds, a screener must identify all objects in each bag and make many decisions within this short period and be able to do it hundreds of times during a shift. Having the best image available to the screener is of paramount importance.

Current image processing techniques include:

- histogram equalization: in this technique, the histogram of the image is modified by using an automatic, mathematically derived algorithm that causes darker regions in an image to appear brighter. However, this method suffers from saturation in the bright regions.

- high-pass filtering: in this technique, the high-frequency features, i.e., the edge features, are highlighted. This makes it easier to see the finer detail in the image and eliminates some of the impact of illumination changes.

- unsharp masking: in this technique, the high-pass filtered image is added back to the original image to enhance the edge features yet keep the original image context. This is typically not an automatic technique and it can take the operator a few tens of seconds to obtain the desired results. 
- other methods of histogram modification: there are several other histogram modification techniques, some automatic and others not. Most popular of these is the automatic enhancement technique known as autolevels. Autolevels uses a single user defined parameter to enhance images. Though autolevels produces good results for illumination changes, it fails when the image has a wide dynamic range.

Although these methods may increase contrast and improve some imagery, their ability to be consistently effective is quite limited. Figure 1 shows an image of a typical piece of carry-on baggage acquired with a computerized radiography system. The images are not of a poor quality but, because of the fact that some objects lie on top of other objects, all the details for all the objects cannot be seen clearly. Two versions of the scene are shown. One acquired at twice the exposure as the other. Each of these exposures has advantages over the other. The darker image displays the light areas very well without saturations while it hides some detail in the dark areas. The lighter image shows much more detail in the dark areas but at the expense of increased saturations of the already bright regions. Some combination of these two images or more would be needed to display the full dynamic range of the scene were it not for the availability of some form of image processing.

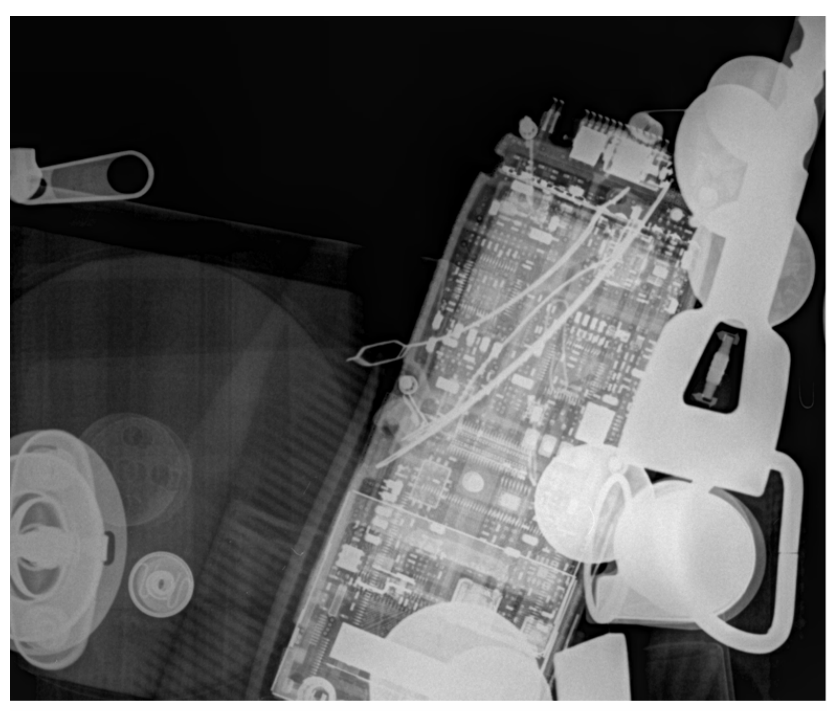

a

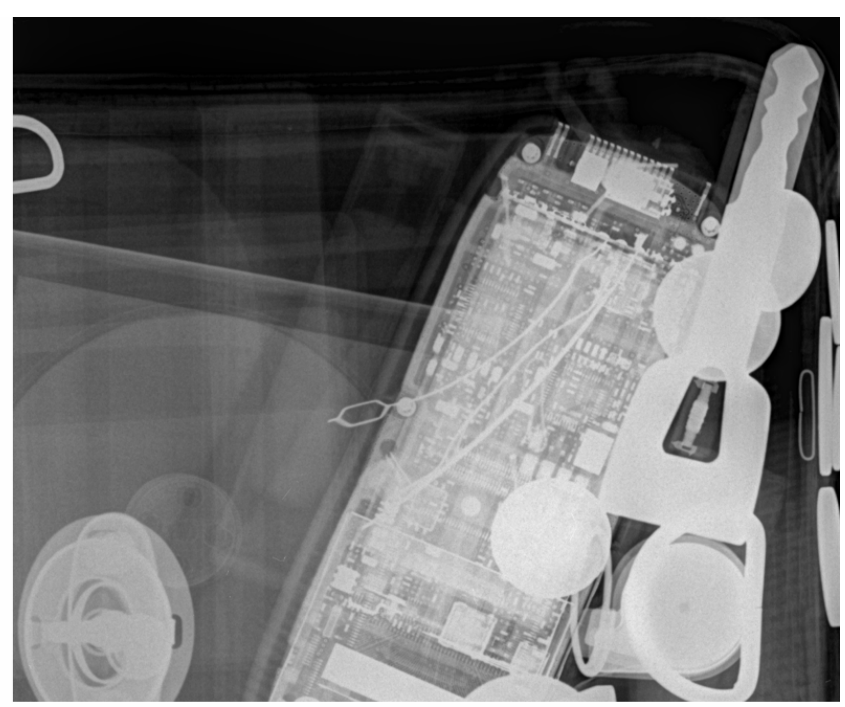

b

Figure 1. Example of typical carry-on baggage: a) acquired with computer radiography system, b) same object acquired at twice the exposure.

Figure 2 shows a cropped section of Figure 1 and several processed results. The processing was done using both automatic and manual conventional image enhancement techniques. Histogram equalization and autolevels are automatic techniques that base their processing on the scene histogram, whereas curves is a manual technique that modifies the relationship between the intensity levels that are displayed and the intensity levels that are acquired. Both high-pass filtering and unsharp masking modify the edge-features in the image so both are spatially non-linear. While all of these techniques do offer some improvement on the overall contrast and brightness, they only provide marginal dynamic range compression over the entire image and very little sharpening if any at all. Many areas of high brightness are saturated completely and the images are still not as sharp as they could be. Additionally, though these techniques may work well with some images, they don't always work well on most images. Hence their overall effectiveness is rather limited.

\section{RETINEX ENHANCEMENTS}

Although most of the wide dynamic range data is available in each image, the image display is not capable of displaying it properly. The wide dynamic range of the original image has to be reduced in order to match the narrow dynamic 


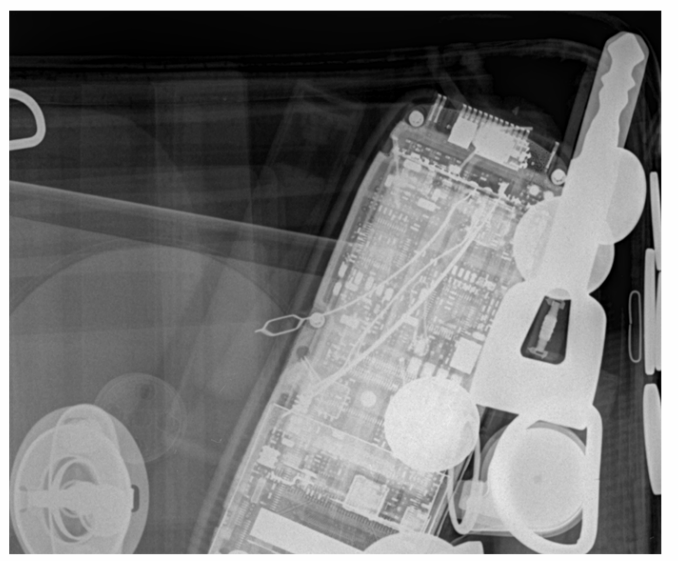

Original

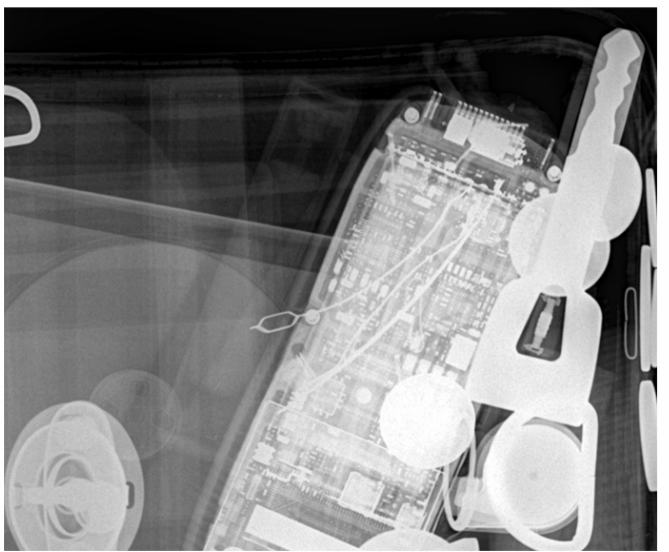

Auto levels

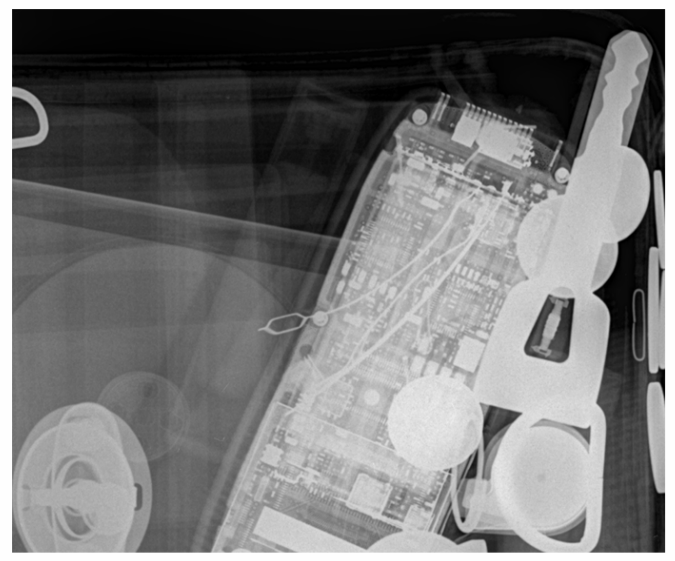

High pass filter

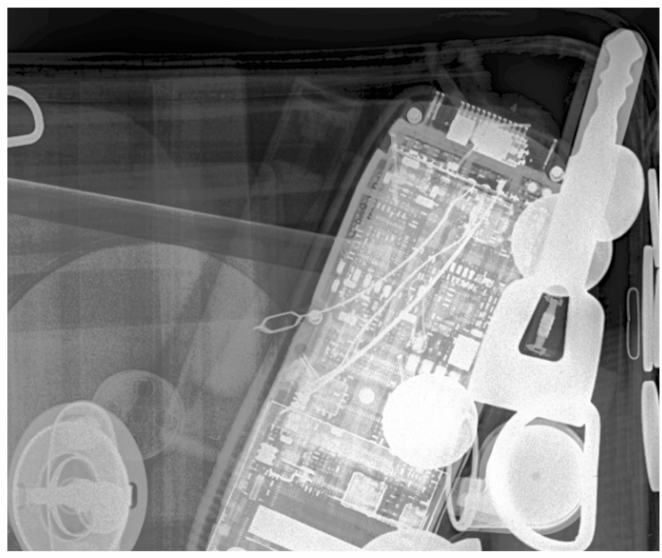

Histogram equalization

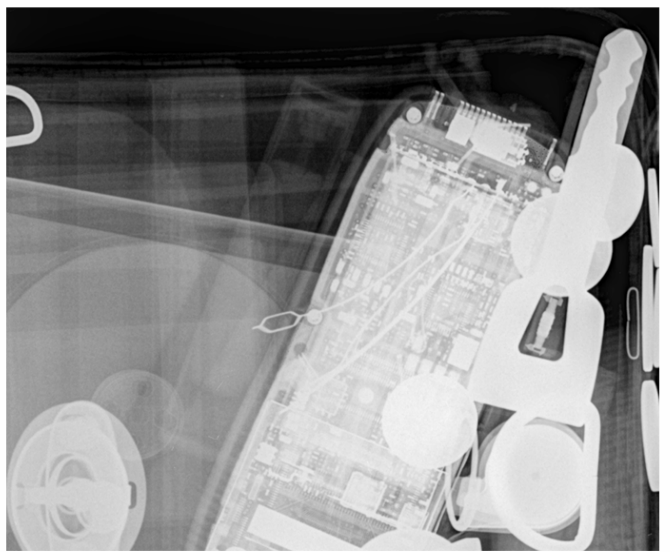

Curves modification

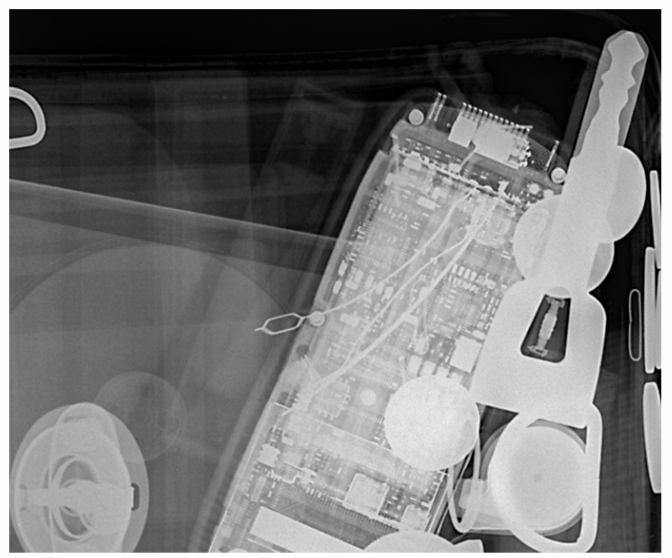

Unsharp mask

Figure 2. Various traditional image processing techniques applied to the radiograph shown in top-left. Whereas each method is successful in enhancing the overall image, none of them quite succeeds in bringing out the contrast in the fine detail such as that seen across the electronic circuit board. The most successful enhancement for this radiograph is, perhaps, histogram equalization. 
range of the display. Traditional image processing techniques such as those mentioned above are typically used to compress the dynamic range of the image so that it makes a better fit with the display. Many of these techniques are capable of performing quite well on a limited set of images but fail considerably with other images. The Retinex is a multi-scale, non-linear operation that analyzes each pixel in an image in the context of its surrounding pixels. This spatial dependency provides strong local contrast enhancement that brings out the details in the dark areas in an image yet areas that are already bright are not saturated. Additionally, the Retinex provides intrinsic sharpening. Overall, the scene is brighter, sharper and has more contrast. And because this enhancement operates on every pixel independently, i.e., it is context-dependent, it works very well on far more images than any other method of processing ${ }^{6}$. Once the optimal settings are determined for a particular class of images (consumer, X-ray, haze, etc.) the processing is completely automatic and requires no user input.

In Figure 3, a cropped version of the image in Figure 2 is examined more closely by comparing the Retinex with the method in Figure 2 which appeared to perform the best with this image: histogram equalization. The Retinex enhancement shows a marked increase in contrast over the entire image but more especially in areas of fine detail. Note that the electronic components of the wireless phone are much easier to distinguish in the Retinex enhancement. The increased sharpness of the Retinex enhancement makes the contacts on the phone much more visible and the hair clip stands out as a separate object. The boundaries of the purse almost disappear completely in the histogram equalization enhancement while they are not lost with the Retinex. The keys are almost completely saturated by histogram equalization while the Retinex merely increases the contrast without saturating. Note that the Retinex enhancement allow you to see completely through the coin at the bottom while in the histogram equalization enhancement the same coin is completely saturated, destroying all traces of information underneath.
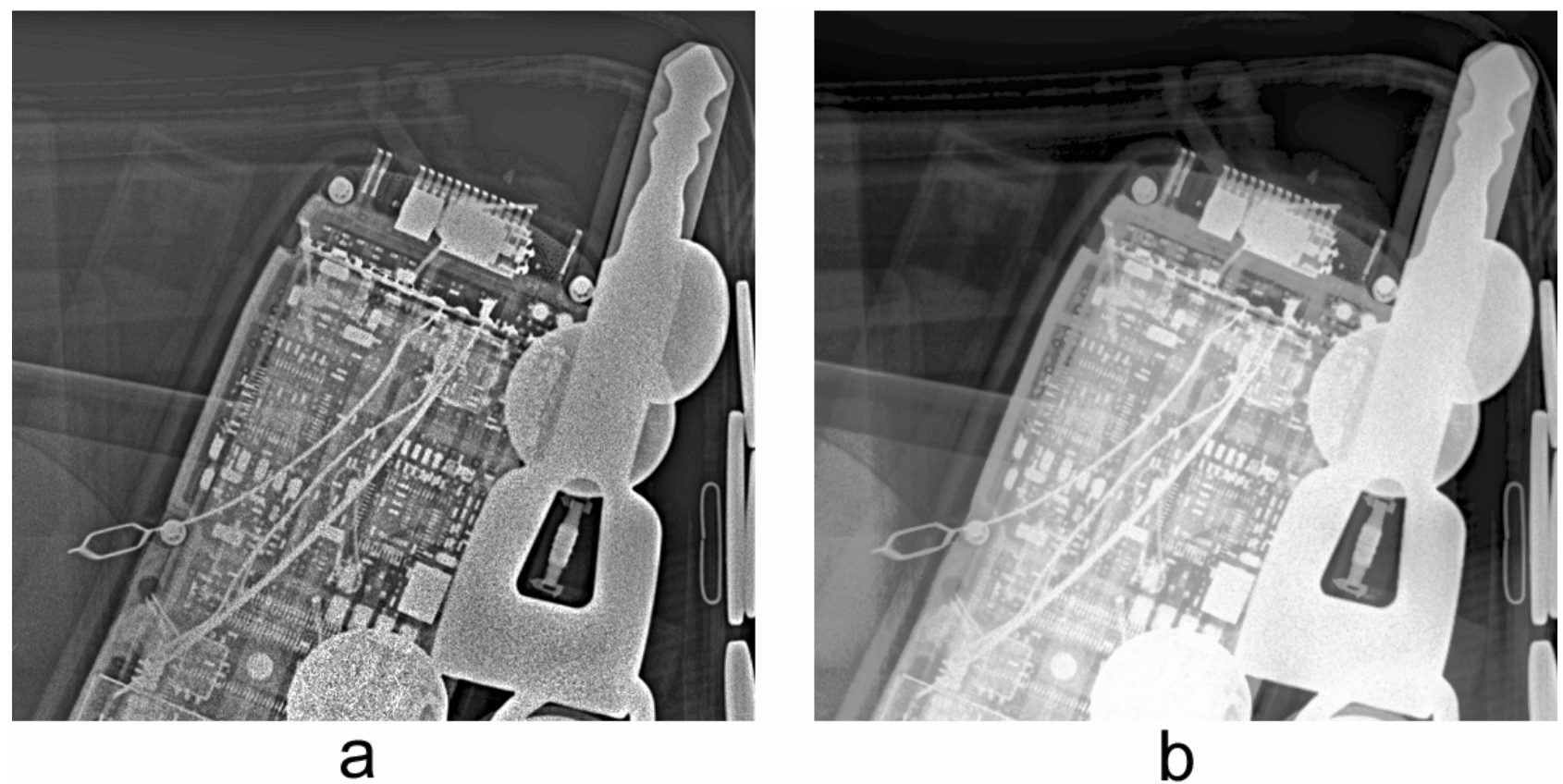

Figure 3. Comparison of Retinex (a) processing with histogram equalization (b).

Although this processing is in current commercial use in software form, it has been demonstrated in hardware and is being developed further in preparation for real-time video applications in support of NASA's Aviation Safety Program. The Retinex works equally well with color images at any bit-depth as well as with infrared, grayscale, and other forms of monochromatic imagery. The MSR is highly computationally intensive and the primary limiting factor for fast 
processing is the amount of processor memory available for algorithm execution. The reader is referred to Jobson ${ }^{2,3}$ et. al and Hines et al. ${ }^{7}$ for details of the MSR algorithm and the related computation issues.

Most testing with radiographs has been with traditional transmissive X-ray systems; however some examples of backscatter X-rays have been performed with equal success. Figure 4 shows several examples of backscattered X-ray cargo imagery. In each of these cases the Retinex enhancements brought out detail that was not clearly visible in the originals. It is also worth noting that these images have probably already been enhanced in some way which indicates that whatever processing had been done to them was insufficient. It may also be possible that Retinex may be performed on top of other forms of processing. In the top images, the items are not any brighter but they have much greater contrast and the sharpness increase is quite noticeable. Note that in each of the two truck images, more detail is seen in the cabs and engine compartments with the Retinex enhancements. Due to the greatly increased sharpness, the gun in the bottom image is picked out almost immediately.

\section{CONCLUSIONS}

With the burden placed on airport baggage screeners to effectively search for prohibited items while keeping the flow of passengers at a manageable level and the false positives to a minimum, it is critical that the operator be given the best image possible. While traditional methods of image processing built into X-ray systems do provide some enhancement, the integration of Retinex processing can further improve upon the images provided to the screener by greatly increasing contrast, sharpness, and brightness where needed. It can be used for traditional grayscale imagery as well as with false-color, organic detection systems. With Retinex hardware integration, even real-time video can have this state-of-the art processing. The benefit of this processing, either in software or hardware, is the reduction in the amount of time needed for a screener to accurately identify recognizable items as suspect or not. This translates to a decrease in tension between passengers and security personnel, more convenience for the passenger with less time lost waiting in lines, and hopefully better confidence for screeners as well as for passengers that the most effective imaging system is being used for the detection of dangerous items. This confidence ultimately means that the passengers feel safer when traveling.

\section{ACKNOWLEDGEMENTS}

The authors would like to thank Phil May, a former trainer for the Transportation Safety Administration, for his insight into current art of screening procedures and technologies, the radiology staff at Riverside hospital for their cooperation in providing some of the images for this work, and NASA Langley's Aviation Safety Program for their support of this work. 

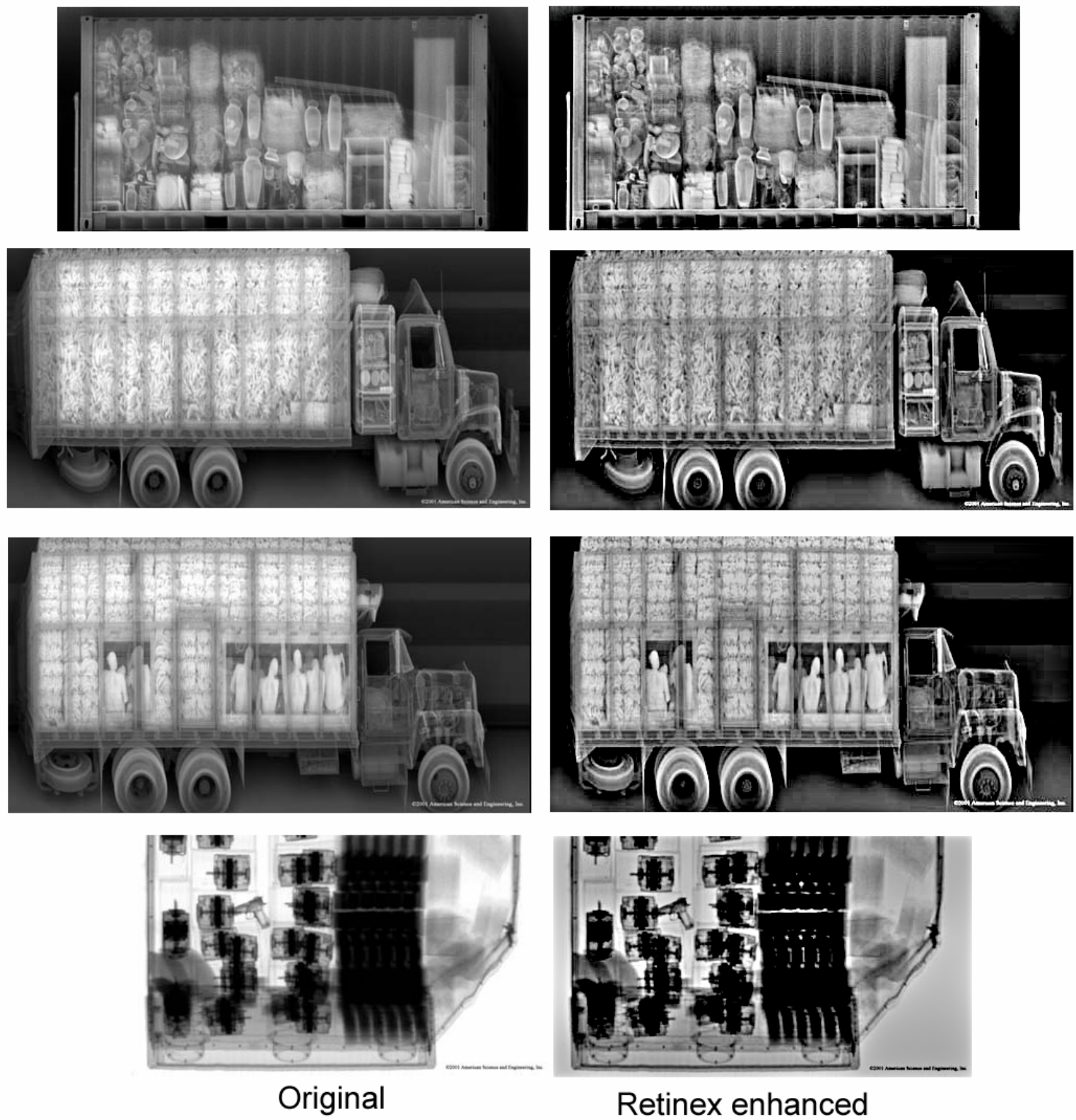

\section{Retinex enhanced}

Figure 4. Retinex enhancements of various images including backscattered X-ray. The original images are shown in the left column and the MSR processed images are shown in the right column. In each case, the increase in contrast and sharpness makes it easier to see the object(s) of interest in the processed image when compares to the original image. For instance, in the images shown in the second row, the processed image provides a much better look inside the engine compartment and the driver's cab than does the original image. Similarly, in the third row, the human-like figures are much easier to see in the MSR-processed image. 


\section{REFERENCES}

[1] Edwin Land, "Recent Advances in retinex theory," Vision Research, 26(1): pp. 7-21, 1986.

[2] Daniel J. Jobson, Zia-ur Rahman, and Glenn Woodell. "Properties and performance of a center/surround Retinex," IEEE Transactions in Image Processing, 6, pp.451-462, March 1997.

[3] Daniel. J. Jobson, Zia-ur Rahman, and Glenn. A. Woodell, "A multi-scale Retinex for bridging the gap between color images and the human observation of scenes," IEEE Transactions on Image Processing: Special Issue on Color Processing, 6, pp. 965-976, July 1997.

[4] Daniel. J. Jobson, Zia-ur Rahman, and Glenn Woodell, "Feature visibility limits in the non-linear enhancement of turbid images," Visual Information Processing XII, Proc. SPIE 5108, (2003).

[5] Zia-ur Rahman, Glenn Woodell, and Daniel J. Jobson, "Retinex Image Enhancement: Application to Medical Images, " presented at the NASA workshop on New Partnerships in Medical Diagnostic Imaging, Greenbelt, Maryland, July 2001.

[6] Z. Rahman, G. A. Woodell, and D. J. Jobson, "A Comparison of the Multiscale Retinex With Other Image Enhancement Techniques," Proceedings of the IS\&T 50th Anniversary Conference, May 1997.

[7] Glenn Hines, Zia-ur Rahman, Daniel Jobson, and Glenn Woodell, "DSP implementation of the multiscale retinex image enhancement algorithm", Visual Information Processing XIII conference, Proc. SPIE 5438, April 2004. 